\section{A TITAN AMONG TONGUE CLEANSERS}

TiTANIUM 2018 is this season's exciting addition to the AMANO range of tongue cleansers.

The TiTANIUM 2018 is made from 100\% titanium - the 22nd element in the periodic table and named after the Greek gods, the Titans.

As befits its name, TiTANIUM is as strong as steel - but less than half the weight (hence why aircraft frames are now made from titanium).

The TiTANIUM 2018 thus offers a supremely smooth and effective

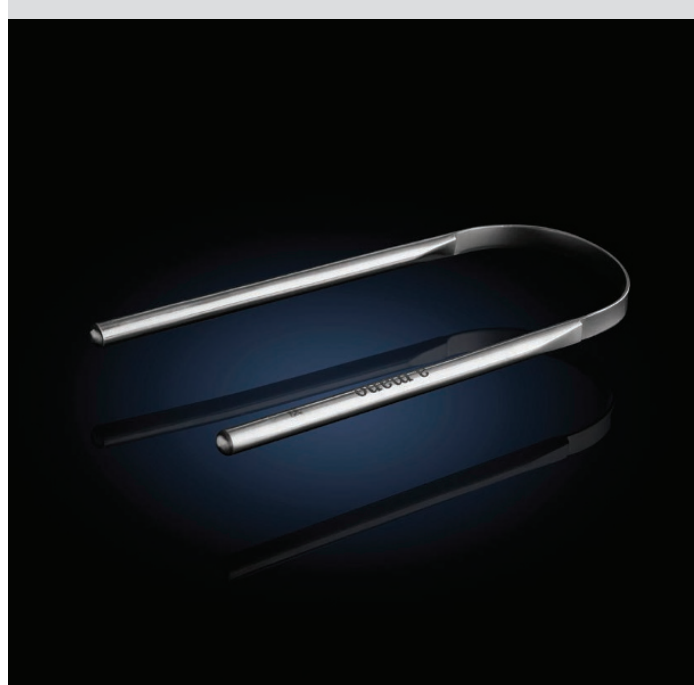

performance - with a slightly lighter feel on the tongue - which some customers might prefer.

The other classic AMANO - the NeoClassic 2017 - is made from a nickel and chrome steel alloy. Some people are allergic to nickel and chrome - so the $100 \%$ pure TiTANIUM 2018 may be a more suitable option for them.

Having designed and made tongue scrapers for 25 years AMANO is keenly aware that the key to 'effectiveness' is not just about how 'sharp' the scraper is. On the contrary, an overly 'sharp' scraper will actually put most people off the routine, whereas a 'comfortable' scraper will mean the user is more relaxed about using a tongue scraper with conviction - and that means also towards the back of the tongue, where oral debris tends to lurk. This means tongue scraping has more of a chance of becoming a new and effective daily routine.

In keeping with its status as the top of the range AMANO, the TiTANIUM 2018 also comes in a metallic presentation case. www.amanotonguecleanser.com

\title{
DON’T LET YOUR PATIENTS RISK A DIY SOS
}

Most of us know the old wives' tales about how you can shortcut your way to better oral health. A very modern concept for the YouTube generation is DIY video guides to filling your own teeth, applying veneers and even giving yourself a whiter smile. But patients need to know that there is a better way.

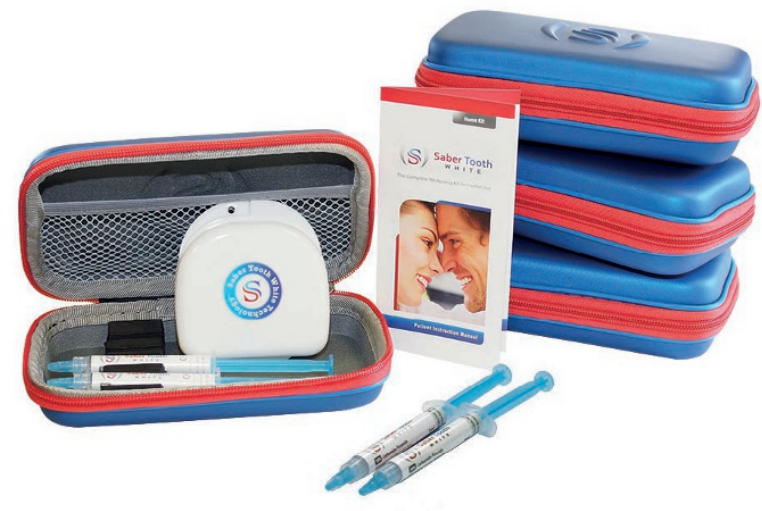

Saber Tooth White Home Kits from CosTech Dental Laboratory bridge the gap between professional-standard products and the comfort and treatment at home.

They provide a high quality, safe, effective and trusted answer to the problem of discoloured teeth at an affordable price.

If you recommend the Saber Tooth White Home Kit to your patients, they will enjoy the physical and psychological benefits of a healthy-looking open smile.

Don't let your patients risk a DIY SOS! Tell them about Saber Tooth White Home Kits today.

Saber Tooth White Home Kits are available from CosTech Dental Laboratory. For more information, visit www.sabertoothwhite.com.
NEW TOOTHBRUSH LAUNCHING IN MAY

In April Philips announced the planned launch of the Philips Sonicare ProtectiveClean toothbrush will take place at the British Dental Conference and Dentistry Show in May 2018.

The new ProtectiveClean range incorporates Sonicare's patented technology - 31,000 brush strokes per minute creating dynamic fluid action which drives fluid forces deep into the interproximal spaces and along the gum line, delivering users a gentle, yet effective clean for healthier gums.

Designed to make good oral health more accessible, the striking ProtectiveClean range has options to suit different price points and includes important features recommended by dental professionals and preferred by patients. Primary among then is a unique pressure sensor technology which alerts users when they are applying too much pressure. A brush head replacement reminder tracks the time and pressure a brush head has been used for alerting patients exactly when to replace their brush head, ensuring the toothbrush is always performing at peak performance.

The Philips Sonicare ProtectiveClean toothbrush is proven to reduce gum disease by up to $100 \%$ and remove up to seven times more plaque than a manual toothbrush.

Visit Philips at the British Dental Conference and Dentistry Show to learn more about the Philips Sonicare ProtectiveClean 4300 and the Philips Sonicare ProtectiveClean 6100

Visit http://www. philips.co.uk/ sonicare.

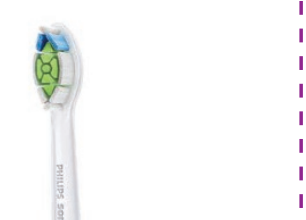

